DIIS - I3A

Universidad de Zaragoza

C/ María de Luna, 1

E-50018 Zaragoza

Spain

Internal Report 2010

Homography-based control scheme for mobile robots with nonholonomic and field-of-view constraints

G. López-Nicolás, N. R. Gans, S. Bhattacharya, C. Sagüés, J. J. Guerrero, and

S. Hutchinson

If you want to cite this report, please use the following reference instead:

G. López-Nicolás, N. R. Gans, S. Bhattacharya, C. Sagüés, J. J. Guerrero, and S. Hutchinson. Homography-based control scheme for mobile robots with nonholonomic and field-of-view constraints. IEEE Transactions on Systems, Man, and Cybernetics: Part B, vol. 10, no. 4, pp. 1115-1127, 2010 


\title{
Homography-Based Control Scheme for Mobile Robots with Nonholonomic and Field-of-View Constraints
}

\author{
G. López-Nicolás, N. R. Gans, S. Bhattacharya, C. Sagüés, J.J. Guerrero and S. Hutchinson
}

\begin{abstract}
In this paper, we present a visual servo controller that effects optimal paths for a nonholonomic, differential drive robot with field-of-view constraints imposed by the vision system. The control scheme relies on the computation of homographies between current and goal images, but unlike previous homography-based methods, it does not use the homography to compute estimates of pose parameters. Instead, the control laws are expressed directly in terms of individual entries in the homography matrix. In particular, we develop individual control laws for the three path classes that define the language of optimal paths: rotations, straight-line segments and logarithmic spirals. These control laws, as well as switching conditions that define how to sequence path segments, are defined in terms of the entries of homography matrices. The selection of the corresponding control law requires the homography decomposition before starting the navigation. We provide a controllability and stability analysis for our system, and give experimental results.
\end{abstract}

Index Terms-Visual control, homography, mobile robot, optimal paths.

\section{INTRODUCTION}

In this paper, we consider the problem of visual servo control of a differential drive vehicle with an on-board monocular vision system. The system must honor nonholonomic constraints imposed by the vehicle kinematics, as well as field-of-view constraints imposed by the camera system. We present a homography-based controller that achieves optimal paths that satisfy these system constraints. To our knowledge, this is the first visual servo control system that guarantees probably optimal paths in the task space. Our control scheme is novel in that it does not use the homography matrix to compute estimates of pose parameters (as pioneered, for example, in [1]). Rather, entries in the homography matrix are used directly in the control law. This reduces the amount of required computation and enhances the system's robustness. One of the key factors enabling our technique is that the qualitative structure of the optimal paths is known a priori [2], reducing the task of the visual servo control system to that of tracking specific curve segments and determining when to

G. López-Nicolás, C. Sagüés and J.J. Guerrero are with the Department de Informática e Ingeniería de Sistemas. Instituto de Investigación en Ingeniería de Aragón. Universidad de Zaragoza, Spain. e-mail: \{gonlopez, csagues, jguerrer\}@unizar.es

N. R. Gans is with the University of Texas at Dallas, Richardson, TX, USA. e-mail: ngans@utdallas. edu

S. Bhattacharya and S. Hutchinson are with the Department of Electrical and Computer Engineering. Beckman Institute for Advanced Science and Technology. University of Illinois, USA. e-mail: \{sbhattac, seth\}@uiuc.edu switch between segments. Thus, control design includes the problem of determining entries for the homography matrix that correspond to subgoals or to the satisfaction of switching conditions. The homography decomposition up to scale is only required to select the appropriate controller and the subgoals definition.

Now in its third decade, visual control, or visual servoing, is an extensive field of research (see, e.g., [3] [4]) in which computer vision is used in the design of motion controllers. Image data can be used directly (image-based methods [5], [6]); it can be used to compute estimates of pose parameters (position-based methods [7], [8]); or various combinations of these approaches can be used (hybrid or partitioned methods [1], [9]). By now, the various trade-offs and shortcomings of these approaches have been investigated and documented (see, e.g., [10], [11] for an overview). On the other hand, some traditional visual control approaches are based on epipolar geometry [12]-[15], but this model is ill conditioned for planar scenes and is problematic with short baseline (i.e., small translation). A good alternative is the homography-based approach [16], but existing approaches usually do not take into account the motion constraints of the platform [17], [18] or require estimation of related depth parameters [19], [20].

Our approach is somewhat different from traditional approaches that seek to design a single control law that guarantees various performance goals. We prefer to combine methods from motion planning (e.g., [21]) with methods from hybrid systems theory to derive a visual servo controller that tracks optimal paths in the Cartesian space using image data, and without resorting to explicit computation of pose. Our proposed homography-based controller is used in a control scheme that is able to maneuver the robot to the goal pose, while keeping the observed target in the camera field-ofview, while following optimal paths in the Cartesian space. For this purpose, we benefit from the work presented in [2], which presents an optimal path scheme for a mobile robot with camera constraints. The optimal paths consist of straight lines and curves that saturate the pan angle of the camera, i.e. the image of the observed target remains on the edge of the camera field-of-view (FOV). In this paper, a controller is presented for each type of path, and as contribution we design a homography-based control scheme to follow these optimal paths. This paper extends the works of [22] and [23] by developing the control scheme covering the entire scene and presenting the controllability and stability analysis. Simulations and real experiments show the feasibility of the 
proposed scheme.

The paper is organized as follows. Section II summarizes the optimal paths scheme to account the field-of-view constraints, and presents the criterion for the selection of the type of optimal paths required. Section III briefly presents the kinematic and imaging models for our system. Section IV presents the control scheme. Sections V and VI study the controllability and stability of our approach, respectively. Simulations and real experiments are given in Section VII.

\section{OPtimal PATHS AND REgIONS}

In this section we briefly summarize the work presented by Bhattacharya et al. in [2] and we present the criteria for deducing which region the robot is in. The work [2] considers the problem of planning shortest paths for differential drive robots whose motion is further constrained by the visual sensor. In particular, they consider the case when the robot must maintain visibility of a fixed landmark using a bodymounted camera with a limited field of view. Kantor and Rizzi [24] and Murrieri et al. [25] have tackled a similar problem without considering issues related to optimality of the paths that are achieved.

The primary result of [2] is that the shortest paths for this system consist of straight-line segments or curves (coined Tcurves) that saturate the sensor viewing angle. The mounted camera is allowed to rotate with respect to the robot body. The camera angle can take values in a closed interval $\left[\psi_{1}, \psi_{2}\right]$ (Fig. 1). The T-curves generated by saturating the camera angle at $\psi_{1}$ are called $\mathrm{T} 1$ curves and the T-curves generated by saturating the camera angle at $\psi_{2}$ are called T2 curves. The $\mathrm{T} 1$ and $\mathrm{T} 2$ curves passing through a point $\mathrm{P}$ are denoted by $T 1_{P}$ and $T 2_{P}$ respectively. This paper replaces a camera which can rotate to keep the target in the center of the image with a fixed camera. The robot can rotate to keep the field of view, and the image of the target is allowed to move from one side of the image to the other (up to a margin in practice). In this case, the range $\left[\psi_{1}, \psi_{2}\right]$ reflects the field-of-view constraint (Fig. 1). Figure 2 shows the final partition of the workspace into regions according to the nature of optimal paths. Refer to [2] for further details.

Before starting navigation, the control needs to ascertain what optimal path is required, based on the target and goal locations (Fig. 2). This will determine the proper control. In the following result, assume that the observed target lies at the origin of the planar coordinate system, denoted $O$. The goal point is denoted by $G$, and without loss of generality we assume $G=\left(r_{G}, 0\right)$ in polar coordinates. The initial point is denoted $S$, with polar coordinates $\left(r_{S}, \theta_{S}\right)$. Table I illustrates the criteria to deduce what region $S$ belongs, based on the value of the ratio $\frac{r_{S}}{r_{G}}$ and the value $\theta_{S}$. The computation of these parameters require the decomposition of the homography once before starting the navigation. SL denotes a straight-line path and $T 1_{G}, T 2_{G}$ denote the T-curves of point $G$. A '-' indicates a smooth transition, while ${ }^{*}$ ' indicates that the robot must rotate to transition from one $\mathrm{T}$-curve to another. The derivation of these criteria is detailed in [22].

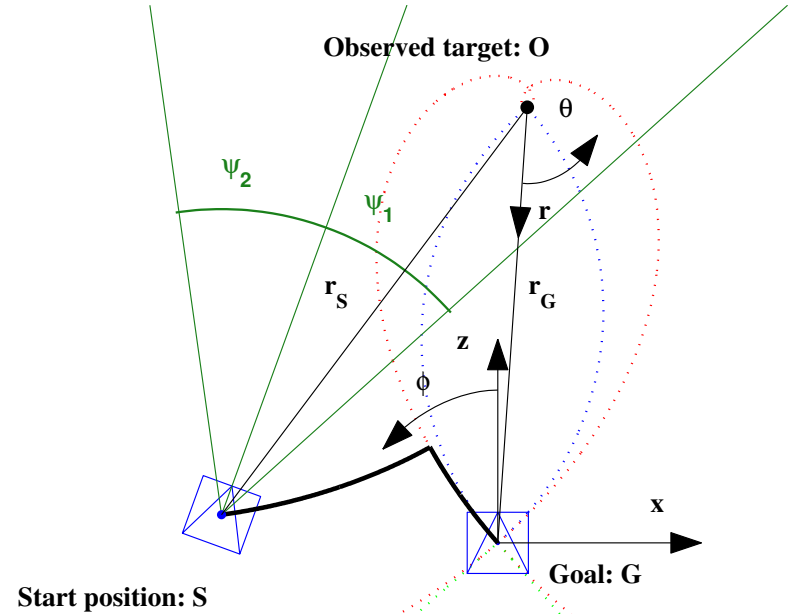

Fig. 1. There are two reference frames. One is defined in the observed target (O) in polar coordinates and is used for the optimal paths definition. The other reference frame is defined in the goal $(\mathrm{G})$ in Cartesian coordinates and is used for the homography definition. The observed target must be kept within the camera field of view, which is constrained by $\left[\psi_{1}, \psi_{2}\right]$.

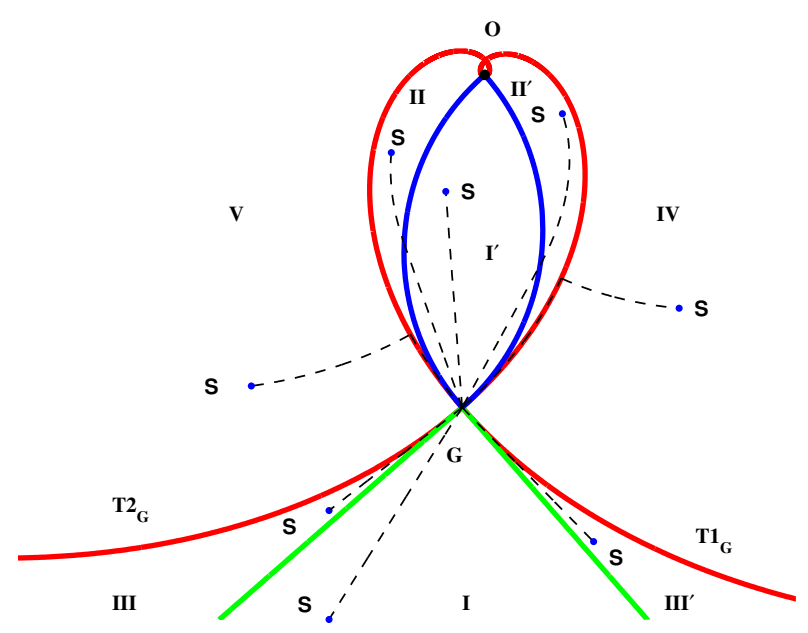

Fig. 2. Regions and shortest paths. The Goal position is $\mathrm{G}$ and the observed target is O. Examples of optimal paths are shown for different initial positions denoted by $\mathrm{S}$.

\section{MODELING}

In this section we define the kinematic model of the robot and describe the geometry of the imaging system. For our application, the desired position of the robot is defined by an image of the observed target taken previously at the goal position. The current and goal images are related by a homography matrix, and it is this matrix that is key to the development of our controller.

We adopt the standard motion model for unicycle robots [21], with the slight variation that we consider motion to occur in the $x-z$ plane instead of the usual $x-y$ plane. This is due to our choice to assign the camera optic axis to be the $\mathrm{z}$-axis of the robot frame. Under this convention, the configuration of the robot system is given by $\mathbf{x}=(x, z, \phi)^{T}$, where $x(t)$ and $z(t)$ are the robot position in the plane, and $\phi(t)$ is the orientation of the robot, expressed as the angle between the robot body 
TABLE I

TYPES OF OPTIMAL PATHS DEPENDING ON THE RATIO $\frac{r_{S}}{r_{G}}$ AND ANGLE $\theta_{S}$ SL DENOTES A STRAIGHT-LINE PATH AND $T 1_{G}, T 2_{G}$ DENOTE THE T-CURVES OF POINT $G$.

\begin{tabular}{|c|c|c|c|}
\hline & $\frac{T_{S}}{r_{G}}$ & $\mid \theta_{S}$ & Type of path \\
\hline \multirow{6}{*}{$\theta \in(-\pi, 0]$} & {$\left[0, \frac{\sin \left(\left|\psi_{1}\right|-\left|\theta_{S}\right|\right)}{\sin \left(\left|\psi_{1}\right|\right)}\right]$} & {$\left[0,\left|\psi_{1}\right|\right]$} & $S L$ \\
\hline & {$\left[\frac{\sin \left(\left|\psi_{1}\right|-\left|\theta_{S}\right|\right)}{\sin \left(\left|\psi_{1}\right|\right)}, e^{\frac{\left|\theta_{S}\right|}{\operatorname{an} \psi_{1}}}\right]$} & {$[0, \pi]$} & $S L-T 1_{G}$ \\
\hline & {$\left[e^{\frac{\mid \theta_{S} S}{\operatorname{an} \psi_{1}}}, e^{\frac{\theta_{S} \mid}{\tan \psi_{2}}}\right]$} & {$[0, \pi]$} & $T 2_{G} * T 1_{G}$ \\
\hline & {$\left[e^{\frac{\left|\theta_{S}\right|}{\tan \psi_{2}}}, \frac{\sin \left(\psi_{2}\right)}{\sin \left(\psi_{2}-\theta_{S} \mid\right.}\right]$} & {$\left[0, \psi_{2}\right]$} & $S L-T 2_{G}$ \\
\hline & {$\left[\frac{\sin \left(\psi_{2}\right)}{\sin \left(\psi_{2}-\theta_{S}\right)}, \infty\right]$} & {$\left[0, \psi_{2}\right]$} & $S L$ \\
\hline & {$\left[e^{\frac{\theta_{S} \mid}{\tan \psi_{2}}}, \infty\right]$} & {$\left[\psi_{2}, \pi\right]$} & $S L-T 1_{G}$ \\
\hline \multirow{6}{*}{$\theta \in[0, \pi)$} & {$\left[0, \frac{\sin \left(\psi_{2}-\theta_{S}\right)}{\sin \left(\psi_{2}\right)}\right]$} & {$\left[0, \psi_{2}\right]$} & $S L$ \\
\hline & {$\left[\frac{\sin \left(\psi_{2}-\theta_{S}\right)}{\sin \left(\psi_{2}\right)}, e^{\frac{-\theta_{S}}{\tan \psi_{2}}}\right]$} & {$[0, \pi]$} & $S L-T 2_{G}$ \\
\hline & {$\left[e^{\frac{-\theta_{S}}{\tan \psi_{2}}}, e^{\frac{-\theta_{S}}{\tan \psi_{1}}}\right]$} & {$[0, \pi]$} & $T 1_{G} * T 2_{G}$ \\
\hline & {$\left[e^{\frac{-\theta_{S}}{\operatorname{an} \psi_{1}}}, \frac{\sin \left(\left|\psi_{1}\right|\right)}{\sin \left(\left|\psi_{1}\right|-\theta_{S}\right)}\right]$} & {$\left[0,\left|\psi_{1}\right|\right]$} & $S L-T 1_{G}$ \\
\hline & {$\left[\frac{\sin \left(\mid \psi_{1}\right)}{\sin \left(\left|\psi_{1}\right|-\theta_{S}\right)}, \infty\right]$} & {$\left[0,\left|\psi_{1}\right|\right]$} & $S L$ \\
\hline & {$\left[e^{\frac{-\theta_{S}}{\operatorname{ann} \psi_{1}}}, \infty\right]$} & {$\left[\left|\psi_{1}\right|, \pi\right]$} & $S L-T 1_{G}$ \\
\hline
\end{tabular}

$z$-axis and the world $z$-axis. This is illustrated in Fig. 1 . The robot has two scalar velocity inputs, linear velocity $v(t)$ and angular velocity $\omega(t)$, where $v$ is in the direction of the robot $z$-axis and $\omega$ is about the robot y-axis (i.e. rotation in the plane).

Consider a set of coplanar points in the world, belonging to a plane $\pi$. Two perspective images of these points can be geometrically linked by a homography $\mathbf{H} \in \mathbb{R}^{3 \times 3}$ [26], [27]. We suppose that the two images are obtained with the same camera, and that one of these images is acquired from the goal pose corresponding to $\mathbf{x}=(0,0,0)^{T}$. The second image is captured at the current pose, which is related to the reference pose by a rotation $\mathbf{R}(t)$ and translation $\mathbf{c}(t)$. The homography $\mathbf{H}$ can be related to camera motion up to scale as

$$
\mathbf{H}=\mathbf{K} \mathbf{R}\left(\mathbf{I}+\mathbf{c} \frac{\mathbf{n}^{T}}{d}\right) \mathbf{K}^{-1},
$$

where $\mathbf{n}=\left(n_{x}, n_{y}, n_{z}\right)^{T}$ is the unit normal of the plane $\pi$ with respect to the reference frame and $d$ is the distance along $\mathbf{n}$ between the plane and the reference position. Both $\mathbf{n}$ and $d$ are constant during the motion since they are defined relative to the fixed reference frame. The matrix $\mathbf{K}$ is the intrinsic camera calibration with focal length $\alpha_{x}$ and $\alpha_{y}$ in pixel dimensions [27]. In practice, we assume that the principal point is in the center of the image and that there is no skew.

The general homography $\mathbf{H}$ has nine elements $h_{i j}$ with $i, j=1,2,3$, where $i$ and $j$ are the row and column index, respectively. Planar motion constrains some elements of $\mathbf{H}$ such that $h_{21}=0, h_{22}=1$ and $h_{23}=0$. Therefore $h_{22} \neq 0$ and we can always fix the scale of the homography matrix normalizing to $h_{22}=1$. Developing expression (1) we obtain the remaining homography elements as:

$$
\left\{\begin{array}{l}
h_{11}=\cos \phi+(x \cos \phi+z \sin \phi) \frac{n_{x}}{d} \\
h_{12}=\frac{\alpha_{x}}{\alpha_{y}}(x \cos \phi+z \sin \phi) \frac{n_{y}}{d} \\
h_{13}=\alpha_{x}\left(\sin \phi+(x \cos \phi+z \sin \phi) \frac{n_{z}}{d}\right) \\
h_{31}=\frac{1}{\alpha_{x}}\left(-\sin \phi+(-x \sin \phi+z \cos \phi) \frac{n_{x}}{d}\right) \\
h_{32}=\frac{1}{\alpha_{y}}(-x \sin \phi+z \cos \phi) \frac{n_{y}}{d} \\
h_{33}=\cos \phi+(-x \sin \phi+z \cos \phi) \frac{n_{z}}{d}
\end{array}\right.
$$

We have chosen to use only $h_{11}(t), h_{13}(t)$ and $h_{33}(t)$ in the formulation of our control laws. In particular, we have chosen not to use $h_{31}(t)$ and $h_{32}(t)$ because of their sensitivity to noise compared with the rest of the elements, given that they are smaller because of the factors $1 / \alpha_{x}$ and $1 / \alpha_{y}$. In human environments, vertical planes are common, therefore we avoid using elements depending directly on $n_{y}$, like $h_{12}(t)$. Moreover, planes in front of the camera are more easily detected, so in general, we can consider that the normal of detected planes will have $n_{z} \neq 0$. Thus, we assume that the plane detected is not parallel to the camera $z$-axis in the goal location. Refer to [28] for details. In what follows, we develop specific control laws via the analysis of $h_{11}, h_{13}$ and $h_{33}$.

\section{Homography-BASEd CONTROL}

As we have seen in Section II, and particularly in Table I, optimal paths are constructed by chaining together a sequence of primitive motions (straight-line segments, T-curves, and rotations). The appropriate control sequence depends on which region the robot is in. The criteria for determining the sequence is presented in Section II. In this section, we present the control algorithm for each of the three kinds of optimal paths: a straight line, a sequence of two T-curves, or a sequence comprising a straight line followed by a T-curve.

\section{A. Control Law for Paths of Type SL}

When the optimal path is a straight line, up to three individual motions are required: (i) rotate in place until the camera points to the goal, (ii) move in a straight line to the goal, (iii) rotate in place to the goal orientation. This is illustrated in Fig. 3(a). In this case, the robot's motion does not require to saturate the sensor viewing angle to reach the goal and, not surprisingly, we arrive at the same optimal path described in [29].

The key point is to establish the conditions that have to be held during each phase of the navigation. We define $\phi_{t}$ as the angular coordinate denoting the angle required to reach the robot location from the $z$-axis, this angle is illustrated in Fig. 3(a) and it is given by $\phi_{t}=\arctan (-x / z)$. When the robot is directed toward the goal (i.e., $\phi=\phi_{t}$ ), its configuration satisfies $x=-z \tan \phi_{t}$. Using this expression in (2) we have, using $h_{11}\left(\phi_{t}\right)$ as example, that

$$
\begin{aligned}
h_{11} & =\cos \phi_{t}+\left(-z \tan \phi_{t} \cos \phi_{t}+z \sin \phi_{t}\right) \frac{n_{x}}{d} \\
& =\cos \phi_{t}+\left(-z \sin \phi_{t}+z \sin \phi_{t}\right) \frac{n_{x}}{d}=\cos \phi_{t} .
\end{aligned}
$$

Following the same procedure with the other entries, the particular form of the homography for any such configuration 


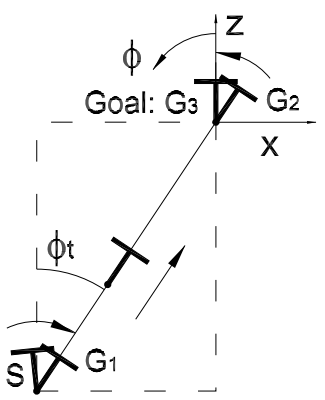

(a) Three steps (Regions I, I')

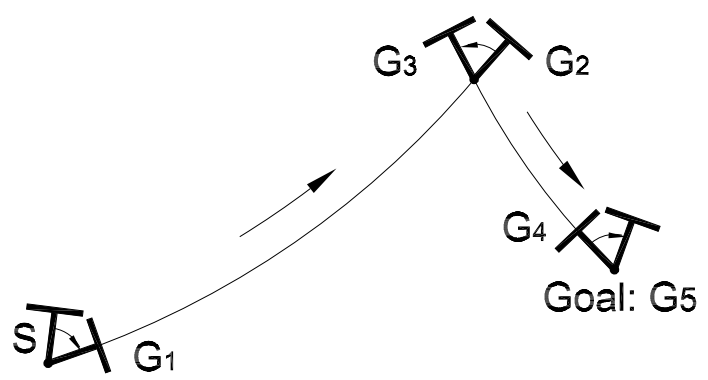

(c) Five steps (Regions IV, V)

Fig. 3. Diagram of the sequence of steps for the different optimal paths.

is given by

$$
\mathbf{H}_{\left(\phi=\phi_{t}\right)}=\left[\begin{array}{ccc}
\cos \phi_{t} & 0 & \alpha_{x} \sin \phi_{t} \\
0 & 1 & 0 \\
\frac{-\sin \phi_{t}}{\alpha_{x}}+\frac{z n_{x} / d}{\alpha_{x} \cos \phi_{t}} & \frac{z n_{y} / d}{\alpha_{y} \cos \phi_{t}} & \frac{\cos ^{2} \phi_{t}+z n_{z} / d}{\cos \phi_{t}}
\end{array}\right]
$$

There is a singularity in the last row of the previous matrix when $\phi_{t}=\pi / 2$. Note that $\phi=\phi_{t}=\pi / 2$ implies that $z=0$, and this situation cannot happen in regions with paths of type SL, preventing the singularity. From the form of the homography at $\phi=\phi_{t}$ we have

$$
h_{11}^{2}+\frac{h_{13}^{2}}{\alpha_{x}^{2}}=1 .
$$

We assume that the intrinsic camera calibration matrix is known and therefore the value of $\alpha_{x}$ is known. Similarly, using (2) at configuration $\mathbf{x}=\left(0,0, \phi_{t}\right)$ (i.e., the robot is at the goal position, but not yet in the goal orientation) the homography matrix is given by

$$
\mathbf{H}_{\left(x=0, z=0, \phi=\phi_{t}\right)}=\left[\begin{array}{ccc}
\cos \phi_{t} & 0 & \alpha_{x} \sin \phi_{t} \\
0 & 1 & 0 \\
\frac{-\sin \phi_{t}}{\alpha_{x}} & 0 & \cos \phi_{t}
\end{array}\right] .
$$

Thus, at the end of the straight-line motion we have $h_{11}=h_{33}$. Finally, when the robot is in the goal pose, the homography is the identity matrix. Sequencing rotation and straight-line motion controllers, we define the following control algorithm:

Step 1: $v=0, \omega=-k_{\omega}^{\prime}\left(h_{11}^{2}+h_{13}^{2} / \alpha_{x}^{2}-1\right)$

Step 2: $v=-k_{v}\left(h_{11}-h_{33}\right), \omega=-k_{\omega}^{\prime}\left(h_{11}^{2}+h_{13}^{2} / \alpha_{x}^{2}-1\right)$

Step 3: $v=0, \omega=-k_{\omega} h_{13}$

being $k_{\omega}^{\prime}, k_{\omega}$ and $k_{v}$ control gains, with $k_{v}>0$ and $k_{\omega}>0$. Before starting the navigation, and from the information previously used to select in which region the robot is, we can also select the sign of $k_{\omega}^{\prime}$. This sign is determined once at the beginning checking $\operatorname{sign}\left(\phi-\phi_{t}\right)$ together with $\operatorname{sign}\left(h_{11}^{2}+h_{13}^{2} / \alpha_{x}^{2}-1\right)$. We need to determine this sign because the global sign of the error function depends on which region the robot is in and the unknown homography plane parameters. The second step is a straight-line motion but we still compute $\omega$ to correct the orientation error due to noise or drift, which cannot be ignored in real situations.

\section{B. Control Law for Paths of Type T1*T2 or T2*T1}

When the optimal path comprises two T-curves, up to five individual motions may be required as defined in [2]: (i) rotate to align the robot's direction with the tangent to a T-curve, (ii) follow the T-curve to a defined subgoal, (iii) rotate to align the robot's direction with an intersecting T-curve, (iv) follow that T-curve to the goal position, (v) rotate in place to the goal orientation. This is illustrated in Fig. 3(c).

For each of these five steps, we define a subgoal in terms of homography parameters. This control law requires the current orientation and the ratio of the robot position up to scale from the homography decomposition [26] to define the subgoals. Denote $h_{13}^{G_{i}}$ and $h_{33}^{G_{i}}$ as desired values of the homography elements at the end of step $i$. We design the controllers as an error function in terms of the homography elements in which their desired values have to be defined. Sequencing rotation and T-curve controllers, we define the following control algorithm:

Step 1: $v=0, \omega=-k_{\omega}\left(h_{13}-h_{13}^{G_{1}}\right)$

Step 2: $v=-k_{v}\left(h_{33}-h_{33}^{G_{2}}\right), \omega=-k_{\omega}\left(h_{13}-h_{13}^{G_{2}}\right)$

Step 3: $v=0, \omega=-k_{\omega}\left(h_{13}-h_{13}^{G_{3}}\right)$

Step 4: $v=-k_{v}\left(h_{11}-h_{33}\right), \omega=-k_{\omega}\left(h_{13}-h_{13}^{G_{4}}\right)$

Step 5: $v=0, \omega=-k_{\omega}\left(h_{13}-h_{13}^{G_{5}}\right)$

being $k_{v}>0$ and $k_{\omega}>0$ the control gains. To design $h_{13}^{G_{i}}$ and $h_{33}^{G_{i}}$, note that from (2), $n_{z} / d$ is a constant and can be solved at the current pose and substituted into the expressions for the subgoal pose. The parameters $h_{13}^{G_{i}}$ and $h_{33}^{G_{i}}$ are then obtained as

$$
\begin{aligned}
& h_{13}^{G_{i}}=\frac{\left(\frac{h_{13}}{\alpha_{x}}-\sin \phi\right)\left(\rho^{G_{i}} \cos \phi^{G_{i}}+\sin \phi^{G_{i}}\right)}{(\rho \cos \phi+\sin \phi) \rho_{z} / \alpha_{x}}+\alpha_{x} \sin \phi^{G_{i}} \\
& h_{33}^{G_{i}}=\frac{\left(h_{33}-\cos \phi\right)\left(-\rho^{G_{i}} \sin \phi^{G_{i}}+\cos \phi^{G_{i}}\right)}{(-\rho \sin \phi+\cos \phi) \rho_{z}}+\cos \phi^{G_{i}}
\end{aligned}
$$

where the ratios $\rho(t)=x / z, \rho^{G_{i}}=x^{G_{i}} / z^{G_{i}}$ and $\rho_{z}(t)=z / z^{G_{i}}$ are defined to ease notation. The current robot orientation is $\phi$ and the desired orientation in each step is $\phi^{G_{i}}$. Note that (6) and (7) contain time varying terms, however these terms are constrained in such a way that $h_{13}^{G_{i}}$ and $h_{33}^{G_{i}}$ remain constant. The elements $h_{13}$ and $h_{33}$ are known from the homography and, through its decomposition [26], [30], $\phi$ and $\rho$ are also known. Notice that the homography decomposition is only used for the subgoal definition. Values for $\phi^{G_{i}}, \rho^{G_{i}}$ and $\rho_{z}^{G_{i}}$ must be defined to obtain the values of $h_{13}^{G_{i}}$ and $h_{33}^{G_{i}}$ for each subgoal. We now discuss how to do this.

1) Subgoals $G_{1}, G_{3}$ and $G_{5}$ : The steps 1 and 3 consist in rotating the robot until it is oriented tangent to the corresponding T-curve. Since $v=0$ in these steps, $\rho^{G_{1}}=\rho^{G_{3}}=\rho$ and 


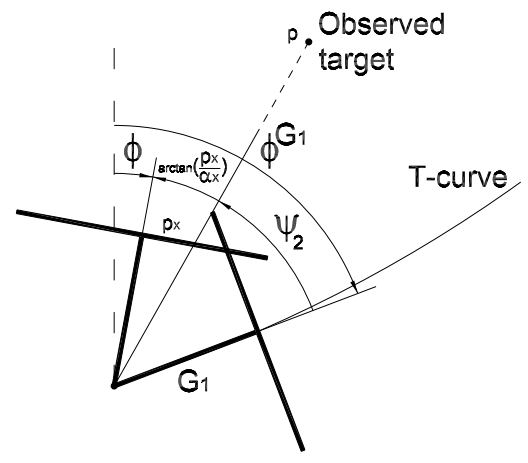

Fig. 4. Angles involved in the computation of $\phi^{G_{1}}$ (8). Two cameras are drawn, one at a current position and the other at the desired subgoal. The desired orientation in $G_{1}$ implies the robot to be on the T-curve.

$\rho_{z}=1$ in (6) and (7). The desired orientations are

$$
\begin{aligned}
\phi^{G_{1}} & =\phi-\arctan \left(\frac{p_{x}}{\alpha_{x}}\right)-\psi_{2} \\
\text { and } \phi^{G_{3}} & =\phi-\arctan \left(\frac{p_{x}}{\alpha_{x}}\right)-\psi_{1},
\end{aligned}
$$

where $p_{x}(t)$ is the $x$-coordinate of a point of the target in the current image and $\left[\psi_{1}, \psi_{2}\right]$ are the limits of the camera field of view. The deduction of $\phi^{G_{1}}$ can be seen graphically in Fig. 4. Note that the time varying terms in (8) and (9) are constrained such that $\phi^{G_{1}}$ and $\phi^{G_{3}}$ remain constant. In step 5 the robot performs a rotation to converge the homography matrix to identity, therefore $h_{13}^{G_{5}}=0$.

2) Subgoal $G_{2}$ and $G_{4}$ : T-curves are followed to reach subgoals $G_{2}$ and $G_{4}$. The desired orientations $\phi^{G_{2}}$ and $\phi^{G_{4}}$ are computed as (8) and (9) respectively. These expressions define not only the final desired orientation at the end of $G_{2}$ and $G_{4}$ but its evolution, in such a way that the robot moves in the T-curve. Given that the orientation correction is responsible for keeping the robot moving in the T-curve, we have that $\rho^{G_{2}}=\rho^{G_{4}}=\rho$ and $\rho_{z}=1$.

In step 4 the robot moves backwards along a T-curve until it reaches the goal position. During this step, we require $h_{33}$ to converge to $h_{11}$, as seen in (5). This condition determines the velocity of the robot moving along the T-curve. The analytical expressions of the T-curves consist of logarithmic spirals which were deduced in [2] as follows. Let $\left(r_{1}, \theta_{1}\right)$ a point of the T-curve and $(r, \theta)$ a general point on the curve. The differential equation of a planar curve is given [31] as

$$
\frac{1}{r} \frac{d r}{d \theta}=\frac{-1}{\tan \psi} \text {. }
$$

Integrating this expression and taking into account that $\psi$ is constant, we have

$$
\begin{gathered}
\int_{r_{1}}^{r} \frac{1}{r} d r=\int_{\theta_{1}}^{\theta} \frac{-1}{\tan \psi} d \theta, \\
\ln \frac{r}{r_{1}}=\frac{\theta_{1}-\theta}{\tan \psi} .
\end{gathered}
$$

This expression can be written for the case of a T1 and T2 curve with respect to the subgoal location as

$$
\frac{r^{G_{2}}}{r_{1}}=e^{\frac{\theta_{1}-\theta^{G_{2}}}{\tan \psi_{1}}} \text { and } \frac{r^{G_{2}}}{r_{5}}=e^{\frac{\theta_{2}-\theta^{G_{2}}}{\tan \psi_{2}}} .
$$

For $h_{33}^{G_{2}}$, we have at the end of this step $\phi^{G_{2}}=\theta^{G_{2}}-\psi_{2}$, where $\theta^{G_{2}}$ is computed from the equations of the T-curves (10), at the end of the second step as

$$
\theta^{G_{2}}=\frac{\ln \left(r_{1} / r_{5}\right)+\left(\theta_{1} / \tan \psi_{1}-\theta_{5} / \tan \psi_{2}\right)}{1 / \tan \psi_{1}-1 / \tan \psi_{2}},
$$

where $\left(r_{1}, \theta_{1}\right)$ designate the initial position and $\left(r_{5}, \theta_{5}\right)$ the goal. The ratio $r_{1} / r_{5}$ (see Fig. 1) can be computed as

$$
\frac{r_{1}}{r_{5}}=\frac{\rho_{15} \cos \left(\gamma_{5}\right)+\sin \left(\gamma_{5}\right)}{\rho_{15} \sin (\beta)-\cos (\beta)},
$$

with $\gamma_{5}=\arctan \left(\frac{p_{x 5}}{\alpha_{x}}\right), \beta=\pi / 2+\psi_{2}-\theta_{15}, \rho_{15}=\rho^{G_{1}}$ and $\theta_{15}$ given by $\theta_{15}=\theta_{5}-\theta_{1}=\gamma_{1}-\gamma_{5}-\phi_{15}$, where $\gamma_{1}=\arctan \left(\frac{p_{x 1}}{\alpha_{x}}\right)$, and $\phi_{15}$ is the relative orientation between initial and goal position obtained from the homography decomposition [30]. The scalars $p_{x 1}$ and $p_{x 5}$ are the $x$-coordinates of a point on the target in the initial and goal images respectively. We have fixed our reference at $\theta_{5}=0$.

Assumption 4.1: For $h_{33}^{G_{2}}$ we can compute neither $\rho^{G_{2}}$ nor $\rho_{z}$ because we need the value of $\rho$ in the subgoal position of step 2 which can only be known when this position is reached. The orientation velocity $\omega$ is computed without any approximation ensuring that the robot follows the T-curve. Therefore, it is known that $\rho \rightarrow \rho^{G_{2}}$ and $\rho_{z} \rightarrow 1$ as the system evolves through the T-curve, and it is ensured that eventually $\rho=\rho^{G_{2}}$ and $\rho_{z}=1$ in the subgoal location. Then, we use the following approximation instead: $\rho^{G_{2}} \simeq \rho$ and $\rho_{z} \simeq 1$, with $\rho$ defined in the current position.

This approximation in $h_{33}^{G_{2}}$ (7) simplifies the computation of the control velocity $v$ for subgoal $G_{2}$ allowing to control properly the forward distance to the corresponding subgoal. The approximation affects $v$ in a scale factor while the desired orientation is independently obtained by means of $\omega$, guaranteing that the subgoal location is reached. The validity of this assumption is demonstrated in the stability analysis and supported by the experimental evaluation. Angles $\psi_{1}$ and $\psi_{2}$ can be exchanged in the previous equations depending on the type of optimal path selected (Section II).

\section{Control Law for Paths of Type SL-T1 or SL-T2}

When the optimal path comprises a T-curve followed by a straight line, up to four individual motions may be required: (i) rotate to align the robot's direction with the tangent to a T-curve, (ii) follow the T-curve to a defined subgoal, (iii) follow straight line to the goal position, (iv) rotate in place to the goal orientation. This is illustrated in Fig. 3(b). Sequencing rotation, straight-line motion and T-curve controllers we obtain the following control algorithm:

Step 1: $v=0, \omega=-k_{\omega}\left(h_{13}-h_{13}^{G_{1}}\right)$

Step 2: $v=-k_{v}\left(h_{33}-h_{33}^{G_{2}}\right), \omega=-k_{\omega}\left(h_{13}-h_{13}^{G_{2}}\right)$

Step 3: $v=-k_{v}\left(h_{11}-h_{33}\right), \omega=-k_{\omega}^{\prime}\left(h_{11}^{2}+h_{13}^{2} / \alpha_{x}^{2}-1\right)$

Step 4: $v=0, \omega=-k_{\omega}\left(h_{13}-h_{13}^{G_{4}}\right)$

being $k_{v}, k_{\omega}$ and $k_{\omega}^{\prime}$ control gains defined as in the previous sections. The desired values of the homography elements $h_{13}^{G_{i}}$ and $h_{33}^{G_{i}}$ for each subgoal are computed using (6) and (7). The definition of the values of $h_{13}^{G_{i}}$ and $h_{33}^{G_{i}}$ for subgoals $G_{1}, G_{3}$ and $G_{4}$ can be deduced directly from the controllers of the previous 
sections. For Subgoal $G_{2}$ the robot moves following a T-curve, in this case the desired value of $\phi^{G_{2}}$ in (6) is computed as (9). For $h_{33}^{G_{2}}$, we have $\phi^{G_{2}}=\theta^{G_{2}}+\psi_{1}$, where the value of $\theta^{G_{2}}$ characterizes the intersection between the T-curve and the straight-line path. Next, the value of $\theta^{G_{2}}$ is deduced. We have defined $\left(r_{1}, \theta_{1}\right)$ as the initial position, $\left(r_{4}, \theta_{4}\right)$ as the goal and $\left(r_{2}, \theta_{2}\right)$ as the intersection of the T-curve with the straightline path (see Fig. 3(b)). The subgoal $G_{2}$ lies on a T-curve, therefore the next expression holds

$$
\frac{r_{2}}{r_{1}}=e^{\frac{\theta_{1}-\theta_{2}}{\tan \psi_{2}}}
$$

The sine rule of the triangle generated by the goal position, the observed target and the intersection gives

$$
r_{2}=r_{4} \frac{\sin \left(\theta_{4}-\theta_{2}+\psi_{1}\right)}{\sin \left(\psi_{1}\right)} .
$$

From (12) and (13) we conclude that

$$
r_{2}=r_{1} e^{\frac{\theta_{1}-\theta_{2}}{\tan \psi_{2}}}=r_{4} \frac{\sin \left(\theta_{4}-\theta_{2}+\psi_{1}\right)}{\sin \left(\psi_{1}\right)} .
$$

where $\theta_{2}$ is the value to find, $\theta_{4}$ is fixed to zero as reference and, from decomposition of the homography we know the ratio $r_{4} / r_{1}$. We want to find the value of $\theta_{2}$, which is bounded by $\left(\theta_{1}, \theta_{4}\right)$. From (14) we have a single 1-dimensional nonlinear equation $f\left(\theta_{2}\right)=0$ which can be solved numerically to $\theta_{2}$.

\section{Controllability Analysis}

In this section we study the controllability of the system when following the optimal paths scheme (Section II) to account field-of-view constraints. The controllability of the homography-based control (Section IV) is also presented.

\section{A. Controllability of the System}

Here, we prove that the differential drive vehicle is controllable when constrained to drive on T-curves and straightline paths that keep the observed target in the field of view. The kinematics of a differential drive vehicle [21] can be expressed with the origin fixed in the observed target, the state of the system denoted as $\left(x_{w}(t), z_{w}(t), \phi(t)\right)^{T} \in \mathbb{R}^{3}$, and $(v, \omega)^{T} \subset \mathbb{R}^{2}$. We describe the position and orientation of the robot in terms of the subgroup of planar motion $S E(2)$ of the Euclidian group in $\mathbb{R}^{3}$. Expressing the kinematics equations in polar coordinates gives

$$
\left(\begin{array}{c}
\dot{r} \\
\dot{\theta} \\
\dot{\phi}
\end{array}\right)=\left(\begin{array}{c}
\cos (\phi-\theta) \\
\frac{1}{r} \sin (\phi-\theta) \\
0
\end{array}\right) v+\left(\begin{array}{l}
0 \\
0 \\
1
\end{array}\right) \omega,
$$

where $r(t)=\sqrt{x_{w}^{2}+z_{w}^{2}}$ and $\theta(t)=-\operatorname{atan}\left(z_{w} / x_{w}\right)$. Without loss of generality we associate the origin for $\theta$ with the $z$-axis of the observed target reference frame.

It is known that a driftless system is small-time controllable if and only if the vector space spanned by the family of vector fields available to the system, along with their Lie brackets, is of full rank everywhere. This is known as the Lie Algebra Rank Condition (LARC) or Chow's Theorem [32][34]. It can be shown that the differential drive vehicle is smalltime controllable given the equation of motion (15) [33]. We now explore the case when the differential drive vehicle is constrained to straight lines, pure rotation, and the available Tcurves. Next we define $\left\{\mathbf{f}_{1}, \mathbf{f}_{2}, \mathbf{f}_{3}, \mathbf{f}_{4}\right\}$ where each $\mathbf{f}_{i},(i=1 . .4)$ is a smooth vector field on $\mathbb{R}^{3}$.

To keep a landmark at the origin within the field of view, the robot must be pointed toward the landmark. This adds a constraint

$$
\phi-\psi=\theta-\pi,
$$

where $\psi(\mathrm{t})$ is the angle between the optical axis of the camera and the observed target image projection. If $\psi \in\left[\psi_{1}, \psi_{2}\right]$ then a point at the origin is visible in the image.

If the robot is moving along a T-curve, then $\psi=\psi_{1}$ or $\psi=$ $\psi_{2}$ (i.e. $\psi$ is constant) and $\dot{\phi}=\dot{\theta}$. Combining (15) and (16) gives the vector fields $\mathbf{f}_{1}(t)$ and $\mathbf{f}_{2}(t)$ available by following T-curves

$$
\left\{\mathbf{f}_{1}, \mathbf{f}_{2}\right\}=\left\{\left(\begin{array}{c}
-\cos \left(\psi_{1}\right) \\
-\frac{1}{r} \sin \left(\psi_{1}\right) \\
-\frac{1}{r} \sin \left(\psi_{1}\right)
\end{array}\right),\left(\begin{array}{c}
-\cos \left(\psi_{2}\right) \\
-\frac{1}{r} \sin \left(\psi_{2}\right) \\
-\frac{1}{r} \sin \left(\psi_{2}\right)
\end{array}\right)\right\} .
$$

If the robot is moving along a straight line while keeping the origin in the field of view, then $\psi \in\left(\psi_{1}, \psi_{2}\right)$ and $\dot{\phi}=0$. In this case the robot is following a vector field

$$
\mathbf{f}_{3}(t)=\left(\begin{array}{c}
\cos (\phi-\theta) \\
\frac{1}{r} \sin (\phi-\theta) \\
0
\end{array}\right)
$$

being $\phi$ constant. If the robot is rotating in place, such as moving between T-curves, then the robot follows a vector field

$$
\mathbf{f}_{4}(t)=\left(\begin{array}{l}
0 \\
0 \\
1
\end{array}\right)
$$

Together, the vector fields $\mathbf{f}_{1}$ through $\mathbf{f}_{4}$ give the vector space of available velocities for the robot. It can be seen that the span of vector field $\left\{\mathbf{f}_{1}, \mathbf{f}_{2}, \mathbf{f}_{3}, \mathbf{f}_{4}\right\}$ is full rank. The vector field becomes undefined at $r=0$. However this situation can not occur under our control, as this case would require the robot to intersect the observed target. Thus, the system is smalltime controllable, and by following T-curves and straight lines, the robot can be moved to any position in the plane (other than over the observed target), while keeping the origin in the field of view. In fact, the subset $\left\{\mathbf{f}_{1}, \mathbf{f}_{2}, \mathbf{f}_{4}\right\}$ is full rank, so controllability can be proven even in the case that straight-line motions are not allowed. We have also full rank when only one type of T-curve and a straight line are allowed: $\left\{\mathbf{f}_{1}, \mathbf{f}_{3}, \mathbf{f}_{4}\right\}$ or $\left\{\mathbf{f}_{2}, \mathbf{f}_{3}, \mathbf{f}_{4}\right\}$. On the other hand, in the case of straight-line motions, we have that $\left\{\mathbf{f}_{3}, \mathbf{f}_{4},\left[\mathbf{f}_{3}, \mathbf{f}_{4}\right]\right\}$ is full rank with the Lie bracket $\left[\mathbf{f}_{3}, \mathbf{f}_{4}\right]$ given by

$$
\left[\mathbf{f}_{3}, \mathbf{f}_{4}\right]=\left(\begin{array}{c}
\sin (\phi-\theta) \\
-\frac{1}{r} \cos (\phi-\theta) \\
0
\end{array}\right)
$$

\section{B. Controllability of the Homography-based Scheme}

We are also interested in the controllability of $h_{11}, h_{13}$ and $h_{33}$, since the control law is expressed in terms of these elements. We again turn to the LARC to investigate controllability. 
It has been proved that the span of the vector field available to the robot is full rank. The vector fields were expressed in polar coordinates referred to the observed target, but can be converted to Cartesian coordinates referred to the goal position. Besides, the derivatives of $h_{11}, h_{13}$ and $h_{33}$, given in (2), can be expressed as a function of $(\dot{r}, \dot{\theta}, \dot{\phi})^{T}$ by

$$
\begin{aligned}
\left(\begin{array}{c}
\dot{h}_{11} \\
\dot{h}_{13} \\
\dot{h}_{33}
\end{array}\right)=\mathbf{J}_{\mathbf{h r}}\left(\begin{array}{c}
\dot{x} \\
\dot{z} \\
\dot{\phi}
\end{array}\right)=\mathbf{J}_{\mathbf{h r}} \mathbf{J}_{\mathbf{r c}} \mathbf{J}_{\mathbf{c p}}\left(\begin{array}{c}
\dot{r} \\
\dot{\theta} \\
\dot{\phi}
\end{array}\right), \\
\mathbf{J}_{\mathbf{h r}}(t)=\left[\begin{array}{ccc}
\frac{n_{x}}{d} c_{\phi} & \frac{n_{x}}{d} s_{\phi} & -\left(\frac{n_{x}}{d} x+1\right) s_{\phi}+\frac{n_{x}}{d} z c_{\phi} \\
\alpha_{x} \frac{n_{z}}{d} c_{\phi} & \alpha_{x} \frac{n_{z}}{d} s_{\phi} & \alpha_{x}\left(1+\frac{n_{z}}{d} z\right) c_{\phi}-\alpha_{x} \frac{n_{z}}{d} x s_{\phi} \\
-\frac{n_{z}}{d} s_{\phi} & \frac{n_{z}}{d} c_{\phi} & -\left(\frac{n_{z}}{d} z+1\right) s_{\phi}-\frac{n_{z}}{d} x c_{\phi}
\end{array}\right], \\
\mathbf{J}_{\mathbf{r c}}(t)=\left[\begin{array}{ccc}
c_{\phi} & s_{\phi} & -x s_{\phi}+z c_{\phi} \\
-s_{\phi} & c_{\phi} & -x c_{\phi}-z s_{\phi} \\
0 & 0 & 1
\end{array}\right], \\
\mathbf{J}_{\mathbf{c p}}(t)=\left[\begin{array}{ccc}
\frac{x}{\sqrt{x_{w}^{2}+z_{w}^{2}}} & z_{w} & 0 \\
\frac{z}{\sqrt{x_{w}^{2}+z_{w}^{2}}} & x_{w} & 0 \\
0 & 0 & 1
\end{array}\right],
\end{aligned}
$$

where $s_{\phi}=\sin \phi$ and $c_{\phi}=\cos \phi$. Note that the Jacobian matrix $\mathbf{J}_{\text {cp }}$ is full rank everywhere but the origin (over the observed target), where it is undefined. Assuming $\alpha_{x} \neq 0, n_{z} \neq 0$ and $d \neq 0$, the determinant of $\mathbf{J}_{\mathbf{h r}} \in \mathbb{R}^{3 \times 3}$ is equal zero when

$$
\tan \phi=-\frac{n_{x}}{n_{z}} k \pi, k \in \mathbb{Z} .
$$

This condition is true when the optical axis of the camera is parallel to the plane of feature points. If the condition in (21) is met, the rank of $\mathbf{J}_{\mathbf{h r}}$ is 2 , and there exist velocities that will not result in a change in $\left(\dot{h}_{11}, \dot{h}_{13}, \dot{h}_{33}\right)^{T}$. Substituting $\tan \phi=-\frac{n_{x}}{n_{z}}$ into the equation for $\mathbf{J}_{\mathbf{h r}}$ it has nullspace spanning

$$
\mathbf{f}(t)=\left(\begin{array}{c}
-z-\frac{d}{n_{z}} \\
x \\
1
\end{array}\right) \text {. }
$$

It can be seen from (17)-(19) that none of the used vector fields $\mathbf{f}_{1}$ through $\mathbf{f}_{4}$ belong to the nullspace. Under the proposed control laws, the robot can only move along one of the vectors at any time, and linear combinations of the vectors are unavailable (i.e. arbitrary vectors in the span $\left\{\mathbf{f}_{1}, \mathbf{f}_{2}, \mathbf{f}_{3}, \mathbf{f}_{4}\right\}$ ). Thus, we conclude that $\mathbf{J}_{\mathbf{h r}} \mathbf{J}_{\mathbf{r c}} \mathbf{J}_{\mathbf{c p}}\left(\mathbf{f}_{1}, \mathbf{f}_{2}, \mathbf{f}_{3}, \mathbf{f}_{4}\right)$ is full rank and the system $\left(\dot{h}_{11}, \dot{h}_{13}, \dot{h}_{33}\right)^{T}$ is controllable using $\mathbf{f}_{1}$ through $\mathbf{f}_{4}$.

\section{Stability AnAlysis}

In this section the stability of the control scheme presented is analyzed by means of Lyapunov's Direct Method [35] and LaSalle's invariance principle [36], which has been extended to switched systems [37], [38]. Sections VI-A and VI-B study the stability of each elementary controller and Section VI-C addresses the stability of the switched system with respect to the final goal by defining a common Lyapunov function.

The robot position is expressed with the origin fixed in the observed target as stated previously. We define the next terms expressed in polar coordinates to be used in the definition of the Lyapunov functions

$$
V_{r}=\frac{\left(e_{r}^{G_{i}}\right)^{2}}{2}, \quad V_{\theta}=\frac{\left(e_{\theta}^{G_{i}}\right)^{2}}{2}, \quad \text { and } \quad V_{\phi}=\frac{\left(e_{\phi}^{G_{i}}\right)^{2}}{2},
$$

with $e_{r}^{G_{i}}=\left(r-r^{G_{i}}\right), e_{\theta}^{G_{i}}=\left(\theta-\theta^{G_{i}}\right)$ and $e_{\phi}^{G_{i}}=\left(\phi-\phi^{G_{i}}\right)$. The values $r^{G_{i}}, \theta^{G_{i}}$ and $\phi^{G_{i}}$ denote the desired value of the parameter in the subgoal position of each step $\left(G_{i}\right)$. These functions are positive definite given that $V(\mathbf{x})>0$ for all $\mathbf{x} \neq \mathbf{x}^{G_{i}}$ and $V\left(\mathbf{x}^{G_{i}}\right)=0$ (with $V=V_{r}, V_{\theta}, V_{\phi}$ respectively). The derivatives of the terms (23), which are used in the next subsections are

$$
\begin{array}{r}
\dot{V}_{r}=\left(r-r^{G_{i}}\right) v \cos (\phi-\theta) . \\
\dot{V}_{\theta}=\left(\theta-\theta^{G_{i}}\right) \frac{v}{r} \sin (\phi-\theta) . \\
\dot{V}_{\phi}=\left(\phi-\phi^{G_{i}}\right) \omega .
\end{array}
$$

The time varying control terms in all steps are dependent on the homography entries which are functions of class $C^{\infty}$. The following assumptions are used in different parts of the stability analysis reducing the results to local. We refer to the corresponding assumption when used.

Assumption 6.1: From the field-of-view constraints, we have for typical cameras that $|\psi|<\pi / 2$. Notice that usual cameras are well below this constraint. Additionally, from the region definitions and taking into account that the robot has to observe the target in front of it during the navigation, it turns out that $|\phi|<\pi / 2$.

Assumption 6.2: The linear approximation of a function can be given by using its Taylor expansion. Then, the linear approximation of the sinusoidal functions is given as $\sin \phi \approx \phi$ and $\cos \phi \approx 1$. We have also the linearization result $\rho \approx \rho^{G_{i}}$ and $\rho_{z} \approx 1$ around the subgoal, and $e^{x} \approx 1+x$.

\section{A. Stability Analysis for Paths of Type SL}

The stability of the elementary controllers for following straight-line paths defined in Section IV-A is analyzed next.

Proposition 6.3: Rotation. Step toward $G_{1}$. The controller for rotation in step toward $G_{1}$ in straight-line paths is locally asymptotically stable.

Proof: The robot performs a rotation with $v=0$ and then, we define $V=V_{\phi}$. The desired orientation is $\phi^{G_{1}}=\phi_{t}$. Using (4) and (26) we have

$$
\dot{V}=\dot{V}_{\phi}=e_{\phi}^{G_{1}} \omega=-k_{\omega}^{\prime} e_{\phi}^{G_{1}}\left(h_{11}^{2}+h_{13}^{2} / \alpha_{x}^{2}-1\right) .
$$

Where the sign of $k_{\omega}^{\prime}$ has been selected as explained previously. The error function used to compute $\omega$ is a continuous and decreasing function in $\phi$ (or increasing depending on the region in which the robot is and the homography plane parameters) in the interval limited by the field of view constraints and with solution at $\phi=\phi_{t}$. Then, we can guarantee $\dot{V} \leq 0$ given that $\operatorname{sign} \operatorname{sign}\left(e_{\phi}^{G_{1}}\right) \neq \operatorname{sign}\left(-k_{\omega}^{\prime}\left(h_{11}^{2}+h_{13}^{2} / \alpha_{x}^{2}-1\right)\right)$ and the controller is stable in the Lyapunov sense.

The function (27) depends on a quadratic function with solution the desired orientation $\phi_{t}$ and a second solution that can be denoted as $\phi_{s}$. Additionally, $\phi_{t} \pm \pi$ and $\phi_{s} \pm \pi$ are also solutions. We now study these solutions by means of phase plane analysis. The resultant phase portrait of the system using this controller is depicted in Fig. 5. The plots represent a family of system motion trajectories corresponding to various initial conditions. It can be seen that the desired orientation $\phi_{t}$ and $\phi_{t} \pm \pi$ are stable foci and, on the other hand $\phi_{s}$ and $\phi_{s} \pm \pi$ 


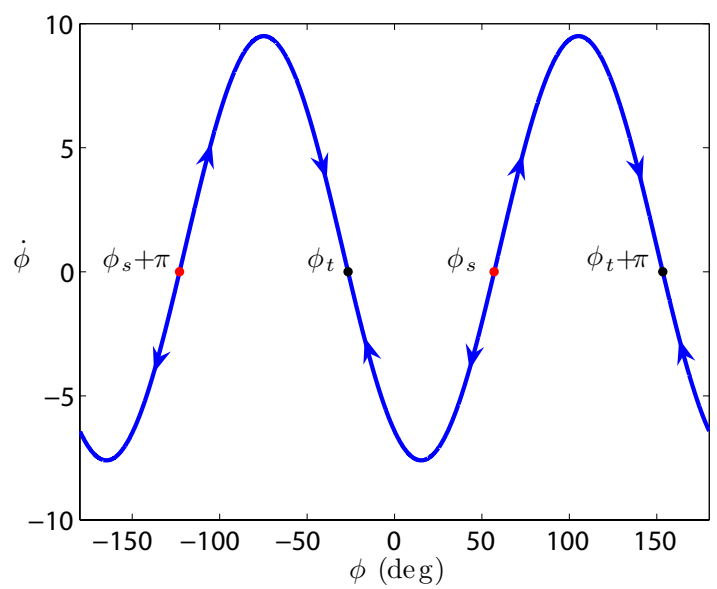

Fig. 5. Phase portrait of $\phi$ using the first controller for following straight-line paths. The desired orientation is $\phi_{t}$.

are unstable foci. Thus, any trajectory starting within the limits of $\phi_{s}$ and $\phi_{s} \pm \pi$ converge to $\phi_{t}$. Any other trajectory out of this region converge to $\phi_{t} \pm \pi$, but this solution is prevented because of field-of-view constraints (Assumption 6.1). So, according to LaSalle the controller is locally asymptotically stable in this region. The application of this controller is constrained to be in regions I and I', with the plane in front of the robot, resulting in an attractive region large enough.

Proposition 6.4: Translation. Step toward $G_{2}$. The controller for translation in step toward $G_{2}$ in straight-line paths is locally asymptotically stable.

Proof: In step 2 the robot moves toward the goal in a straight-line motion with $\phi^{G_{2}}=\phi_{t}$ and we define $V=V_{r}+V_{\phi}$. Thus, from (16), (24) and (26) we have

$$
\dot{V}=\dot{V}_{r}+\dot{V}_{\phi}=-e_{r}^{G_{2}} v \cos (\psi)-e_{\phi}^{G_{2}} \omega .
$$

The velocity given by the control $v=-k_{v}\left(h_{11}-h_{33}\right)$ combined with (3) allows to study analytically its sign:

$$
v=k_{v} \frac{z n_{z}}{d \cos \phi_{t}},
$$

where $k_{v}>0, d>0, n_{z}<0$ and $\cos \phi_{t}>0$. Note that $z$ is the translation from the current to the goal position (Fig. 3(a)). The analysis of the signs (28) and (29) results in $\dot{V} \leq 0$. Analyzing first $\dot{V}_{r}$ and from Assumption 6.1 we have that $\cos \psi>0$. If the robot is behind the goal position we have $e_{r}^{G_{2}}>0$ and $z<0$ (Region I in Fig. 2). Otherwise we have $e_{r}^{G_{2}}<0$ and $z>0$ (Region I'). This gives

$$
\dot{V}_{r}=-e_{r}^{G_{2}} v \cos (\psi)=-\frac{k_{v} n_{z} \cos \psi}{d \cos \phi_{t}} z e_{r}^{G_{2}} \leq 0 .
$$

The analysis of $\dot{V}_{\phi}$ is the same as in (27). Then $\dot{V} \leq 0$ and the controller is stable in the Lyapunov sense. Now we study asymptotic stability by means of LaSalle showing in (28) that $\dot{V}=0 \Leftrightarrow\left(e_{r}^{G_{2}}=0 \wedge e_{\phi}^{G_{2}}=0\right)$. Because of Assumption 6.1 we have $|\psi|<\pi / 2$. Besides, from analysis of the previous controller we have that $\dot{V}_{\phi}=0$ with $\phi=\phi_{t}$ (i.e. $e_{\phi}^{G_{2}}=0$ ) and then, $x=-z \tan \phi$. With $z=0$ and given that $|\phi|<\pi / 2$, we have $x=0$ and then $e_{r}^{G_{2}}=0$. Therefore, the invariant set of the system under this controller is $M=\left\{\mathbf{x} \in \mathfrak{R}^{3}: e_{r}^{G_{2}}=0 \wedge e_{\phi}^{G_{2}}=\right.$ $0\}$ and this controller is locally asymptotically stable.

Proposition 6.5: Rotation. Step toward $G_{3}$. The controller for rotation in step toward $G_{3}$ in straight-line paths is locally asymptotically stable.

Proof: Similar to step 1, we define $V=V_{\phi}$. In this case $\phi^{G_{3}}=0$ and then

$$
\dot{V}=\dot{V}_{\phi}=e_{\phi}^{G_{3}} \omega=-\phi k_{\omega} h_{13}=-k_{\omega} \alpha_{x} \phi \sin \phi \leq 0 .
$$

We have $\dot{V}=0 \Leftrightarrow \phi=(0, \pm \pi)$, but $\phi \neq \pm \pi$ because of fieldof-view constraints. Then $\dot{V}=0 \Leftrightarrow \phi=0$ and through LaSalle this controller is locally asymptotically stable.

\section{B. Stability Analysis for Paths of Type $T 1 * T 2$ or $T 2 * T 1$}

The stability of the elementary controllers for following Tcurves defined in Section IV-B is analyzed next.

Proposition 6.6: Rotations. Steps toward $G_{1}, G_{3}$ and $G_{5}$. The controller for rotation in steps toward $G_{1}, G_{3}$ and $G_{5}$ in T-curves is locally asymptotically stable.

Proof: These steps consist of pure rotations and the Lyapunov functions are defined as $V=V_{\phi}$. From (26) we have

$$
\dot{V}=\dot{V}_{\phi}=e_{\phi}^{G_{i}} \omega=-k_{\omega} e_{\phi}^{G_{i}}\left(h_{13}-h_{13}^{G_{i}}\right), \quad(i=1,3,5) .
$$

For $G_{5}$ the analysis is straightforward. Given that $\phi^{G_{5}}=0$ and $h_{13}^{G_{5}}=0$, (32) reduces to (31). For $G_{1}$ and $G_{3}$, we consider the Assumption 6.2 for $h_{13}^{G_{i}}(6)$ in (32) and then

$$
\begin{aligned}
\dot{V} & =-k_{\omega} e_{\phi}^{G_{i}}\left(h_{13}-\left(h_{13}-\alpha_{x} \phi\right) \frac{\rho+\phi^{G_{i}}}{\rho+\phi}-\alpha_{x} \phi^{G_{i}}\right) \\
& =-k_{\omega} e_{\phi}^{G_{i}} \frac{\phi-\phi^{G_{i}}}{\rho+\phi}\left(h_{13}+\alpha_{x} \rho\right), \quad(i=1,3),
\end{aligned}
$$

and using again Assumption 6.2 with $h_{13}$ (2) we have

$$
\begin{aligned}
\dot{V} & =-k_{\omega} e_{\phi}^{G_{i}} \frac{\phi-\phi^{G_{i}}}{\rho+\phi}\left(\alpha_{x} \phi+\alpha_{x}(\rho+\phi) \frac{z n_{z}}{d}+\alpha_{x} \rho\right) \\
& =-k_{\omega} \alpha_{x}\left(e_{\phi}^{G_{i}}\right)^{2}\left(\frac{z n_{z}}{d}+1\right)<0, \quad(i=1,3) .
\end{aligned}
$$

The plane is in front of the robot and then $-1 \leq n_{z}<0$. If $z \leq 0$ then $\left(z n_{z} / d+1\right)>0$. Otherwise $z>0$ and, given that the robot cannot be behind the plane, we have $d>z$ and $\left(z n_{z} / d+1\right)>0$. Therefore. $\dot{V}<0$ and $\dot{V}=0 \Leftrightarrow\left(e_{\phi}^{G_{i}}\right)^{2}=0$ in (34) and the controller is locally asymptotically stable.

Proposition 6.7: T-curves. Steps toward $G_{2}$ and $G_{4}$. The controller for T-curves in steps toward $G_{2}$ and $G_{4}$ is locally asymptotically stable.

Proof: In these steps we have the Lyapunov candidate function $V=V_{r}+V_{\theta}+V_{\phi}$, so

$$
\dot{V}=\dot{V}_{r}+\dot{V}_{\theta}+\dot{V}_{\phi}=-e_{r}^{G_{i}} v \cos \psi-e_{\theta}^{G_{i}} \frac{v}{r} \sin \psi+e_{\phi}^{G_{i}} \omega,
$$

with $(i=2,4)$ and $(v, \omega)$ from the definition of the control that follows T-curves in Section IV-B. The analysis of $\dot{V}_{\phi}$ in steps 2 and 4 is similar to steps 1 or 3 . Note that the desired orientation varies in order to keep the vehicle moving in the T-curve. It can be seen that in this case (34) holds as well. We 
next analyze the sign of $v$ in (35). As $\phi$ is small with standard cameras $\sin \phi \approx 0$ and using $h_{33}^{G_{2}}$ (7) and $h_{33}$ (2), we have

$$
\begin{aligned}
v & =-k_{v}\left(h_{33}-h_{33}^{G_{2}}\right) \\
& =-k_{v}\left(h_{33}-\left(h_{33}-\cos \phi\right) \frac{\cos \phi^{G_{2}}}{\cos \phi}-\cos \phi^{G_{2}}\right) \\
& =-k_{v}\left(\frac{z n_{z}}{d}+1\right)\left(\cos \phi-\cos \phi^{G_{2}}\right) .
\end{aligned}
$$

As in (34), it can be shown that $\left(z n_{z} / d+1\right)>0$. From the definition of T2-curves in region $\mathrm{V}$ or T1 curves in region IV we have $\left(\cos \phi-\cos \phi^{G_{i}}\right)<0$. Being $k_{v}>0$, we have $v>0$ for $G_{2}$. On the other hand, when the robot moves in a T2 curve to $G_{4}$ it can be deduced from Table I that

$$
\frac{r^{G_{4}}}{r}=\frac{\sin \left(\psi_{2}+\phi-\phi_{t}-\theta+\theta^{G_{4}}\right)}{\sin \left(\psi_{2}+\phi-\phi_{t}\right)} .
$$

Working out the previous equation and using the expression of a T-curve (10) we have

$$
\tan \left(\psi_{2}+\phi-\phi_{t}\right)=\frac{\sin \left(\theta-\theta^{G_{4}}\right)}{\cos \left(\theta-\theta^{G_{4}}\right)-e^{\frac{\theta-\theta^{G_{4}}}{\tan \psi_{2}}}},
$$

and using Assumption 6.2,

$$
\tan \left(\psi_{2}+\phi-\phi_{t}\right)=\frac{\theta-\theta^{G_{4}}}{1-1+\frac{\theta-\theta^{G_{4}}}{\tan \psi_{2}}}=\tan \psi_{2} .
$$

So, $\phi=\phi_{t}$ and the sign of the velocity to $G_{4}$ is given by (29) and then $v<0$ (35). We have that $e_{\theta}^{G_{2}}<0, e_{\theta}^{G_{4}}<0$ and $\cos \psi>0$. Considering the robot in region $\mathrm{V}$, if it moves in step 2 in a T1 curve, we have $e_{r}^{G_{2}}>0$, and $\sin \psi<0$. If the robot moves in step 4 in a T2 curve, we have $e_{r}^{G_{4}}<0$ and $\sin \psi>0$, and then $\dot{V}_{r}<0$ and $\dot{V}_{\theta}<0$. Therefore, $\dot{V}$ is semidefinite negative in steps 2 and 4.

Now we study asymptotic stability by means of LaSalle showing that $\dot{V}=0 \Leftrightarrow\left(e_{r}^{G_{2}}=0 \wedge e_{\theta}^{G_{2}}=0 \wedge e_{\phi}^{G_{2}}=0\right)$ (35). It can be seen from the previous analysis that $\dot{V}$ (35) also can be zero in the next situations: $|\psi|=\pi / 2, \psi=0$ or $\phi=\left(\phi^{G_{i}}, \phi^{G_{i}} \pm \pi\right)$. However these situations are prevented by means of Assumption 6.1 and given that the field of view cannot be constrained to zero $(\psi \neq 0)$. Therefore, using LaSalle this controller is locally asymptotically stable.

A proof for a $\mathrm{T} 2$ curve followed by a $\mathrm{T} 1$ curve follows the same logic. The complete system includes a switch from the straight-line controller to the T-curve controller. The stability analysis for Paths of Type SL-T1 or SL-T2 can be deduced similarly from the previous presented analysis.

\section{Stability of the global trajectories}

We have analyzed the Lyapunov functions defined for each individual controller of the control scheme that follows the optimal paths. Next, we analyze a global function for these paths including all the states.

Proposition 6.8: The control scheme combining the individual controllers to obtain the optimal paths is locally asymptotically stable and the goal location is an invariant set.

Proof: Let us consider a common Lyapunov function

$$
V_{g}\left(r_{g}, t\right)=\left(r_{g}-r_{g}^{G}\right)^{2} / 2 .
$$
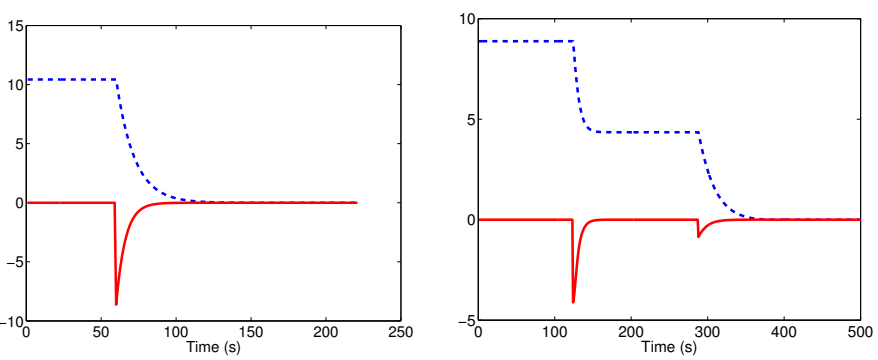

Fig. 6. Examples using the control scheme of the evolution of $r_{g}$ (dashed line) and $\dot{V} g$ (solid line) in the case of a straight line path (a) or path consisting in T-curves (b). The evolution is the result of following the combination of optimal paths as designed.

where $r_{g}>0$ is the radial coordinate of the current position $S$ with the reference fixed in the goal location $G$. The desired value of $r_{g}(t)$ at the goal location is $r_{g}^{G}=0$. The function (40) is positive definite given that $V_{g}>0$ for all $r_{g} \neq 0$ and $V_{g}\left(r_{g}=0\right)=0$. Notice that the desired equilibrium point of the system is defined with $r_{g}=0$ up to rotation in (40), and we have shown from (31) that pure rotation controller is ((locally asymptotically)) stable. We also define the angle $\theta_{g}(t)$ positively measured from $z$-axis anticlockwise with respect to the goal location. By definition, the angle $\theta_{g}$ is bounded and zero when $V_{g}$ is zero. The derivative of (40) yields

$$
\dot{V}_{g}=r_{g} \dot{r}_{g}=r_{g} v \cos \left(\phi-\theta_{g}\right) .
$$

In case of SL trajectories, when the robot is in region I and switches for a straight line we have from (29) that $v>0$ and $\left|\phi-\theta_{g}\right|=\pi$, so $\cos \left(\phi-\theta_{g}\right)<0$. Similar analysis for region I' gives $v<0,\left|\phi-\theta_{g}\right|=0$, and then $\cos \left(\phi-\theta_{g}\right)>0$. Then, the combination of straight-line paths and pure rotations gives $\dot{V}_{g} \leq 0$. An example of the evolution of the function (41) for the SL trajectory can be observed in Fig. 6(a) which shows the negative semi-definiteness.

In case of combination of controllers with T-curves something similar happens. When the robot is in region $\mathrm{V}$ and switches for a T1 curve control we have that $v>0$ decreasing to zero and $\left|\phi-\theta_{g}\right|>\pi / 2$. When the system switches for a T2 curve control we have that $v<0$. Besides, we have that $\cos \left(\phi-\theta_{g}\right)<\pi / 2$ (Fig. 2), otherwise the robot is behind the observed target, so $\cos \left(\phi-\theta_{g}\right)>0$ and then, (41) is definite negative. So, the combination of T-curves and pure rotations gives $\dot{V}_{g} \leq 0$ with respect to the goal. An example of the evolution of the derivative (41) for this case is shown in Fig. 6(b).

The combination of the controllers allows to follow the defined optimal paths [2] and it assures that the system will switch, in finite time, to the following controller when the error, defined in terms of the homography entries, is lower than a threshold. On the other hand, as we have shown, (41) is semi-definite negative. In this case, it is still possible to draw conclusions on asymptotic stability by means of LaSalle. Let us consider $M$ as the invariant set of the system. The goal location is positively invariant if

$$
\mathbf{x}\left(r_{g}=0\right) \in M \Rightarrow \mathbf{x}\left(r_{g}(t)\right) \in M, \forall t \geq 0 .
$$


When the system is in the goal location only orientation is corrected by the control scheme and $\mathbf{x}\left(r_{g}=0\right) \in M$. For any location with $r_{g} \neq 0$ exists a switching control sequence as defined previously that takes the system to the goal location and $\mathbf{x}\left(r_{g} \neq 0\right) \notin M$. Therefore, the largest invariant set is the origin and it is locally asymptotically stable.

\section{EXPERIMENTAL RESULTS}

Simulations and real experiments showing the performance of our proposal are presented in this Section.

\section{A. Simulated Results}

A virtual framework is used by generating random $3 \mathrm{D}$ points in a planar scene. The $3 \mathrm{D}$ points of the scene are projected in the image plane through a virtual camera. The size of the images obtained from the virtual camera is $640 \times 480$ pixels and the field of view is constrained with $\psi_{1}=-26.56^{\circ}$, $\psi_{2}=26.56^{\circ}$. The approach is designed to keep a landmark in the field of view. It is supposed that during the motion the robot can see a plane and estimate the homography. Then, several points are needed, four points in the general case or three points in planar motion [27]. In our simulations we have used more than twenty points to compute the homography when they are noisy. These points to compute the homography usually change as the robot moves. However, the landmark is detected and tracked to be kept in the field of view.

Results of the control following a straight-line path, Tcurves or T-curve plus straight-line path are presented in Fig. 7 , Fig. 8 and Fig. 9 respectively. Two simulations are superposed in each graphic, one without noise and the other adding Gaussian image noise to the points with standard deviation of $\sigma=1$ pixel (thin and thick line respectively). Simulations showing the robot motion together with the evolution of the homography elements used are shown in the video 1 attachment. The simulations show that the controllers proposed to follow the different paths perform properly in spite of image noise.

The control scheme presented needs the camera calibration matrix to be known. In Fig. 10, several results show the performance of the controllers against calibration errors. There are three different types of paths: straight lines, turns and T-curves. The controllers that follow these paths have been tested when there is a calibration error in the focal length or in the coordinates of the principal point. Thus, the real camera configuration is fixed while the values of the camera calibration matrix used in the control are modified. The real values of the camera parameters are $f=6 \mathrm{~mm}$ and $x_{0}=0$. The results obtained are shown in Fig. 10. As expected the experiments show that the final position error increases with the calibration errors. Other sources of error are that we assume that the robot can perform pure rotation on the spot, with the camera centered on the rotation axis, or robot slippage. The robustness of the approach with these sources of uncertainty is tested with a real platform in the next section.

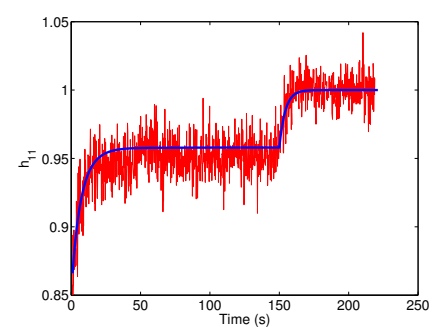

(a) Evolution of $h_{11}$

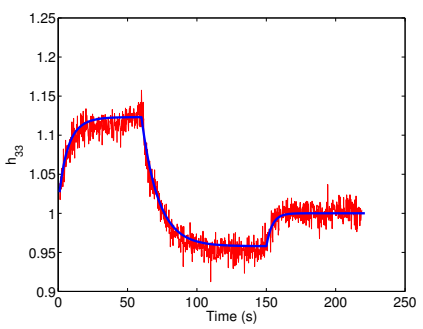

(c) Evolution of $h_{33}$

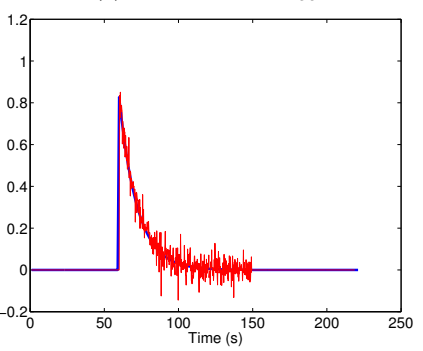

(e) Rotational control error

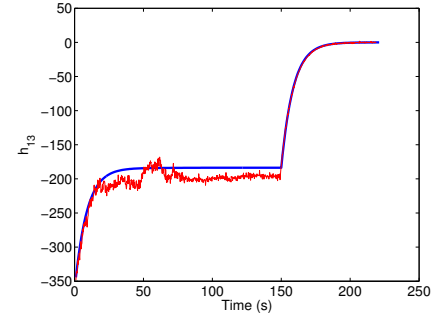

(b) Evolution of $h_{13}$

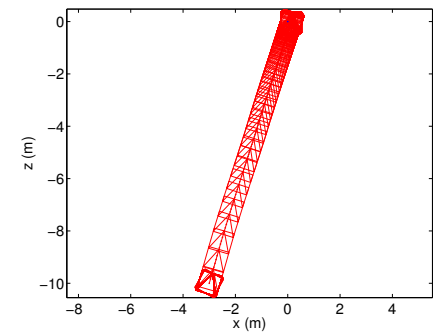

(d) Path with noise

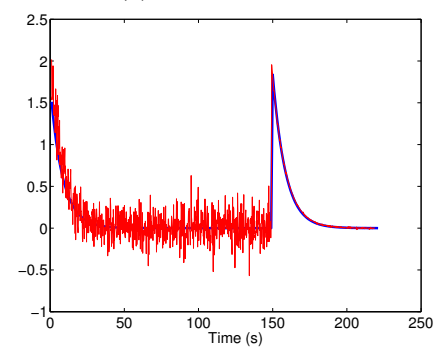

(f) Translational control error
Fig. 7. Simulations following a SL path with and without image noise of $\sigma=1$ pixel. The initial location is $(x, z, \phi)=(-3 \mathrm{~m},-10 \mathrm{~m},-30 \mathrm{deg})$.

\section{B. Real Experiments}

The experimental platform is a Pioneer P3-DX from ActivMedia (Fig. 11). The robot is equipped with a USB camera mounted on top (Logitech QuickCam Communicate STX). The images are acquired at size $640 \times 480$ pixels with field of view of $36.4^{\circ}\left(\psi_{1}=-18.2^{\circ}, \psi_{2}=18.2^{\circ}\right)$. The principal point coordinates are $(331.7143,224.8533)$ pixels and the focal length is $9.1141 \mathrm{~mm}$. The lens distortion coefficients obtained are $K_{1}=-3.799 \cdot 10^{-4}, K_{2}=3.618 \cdot 10^{-6}, P_{1}=-2.251 \cdot 10^{-4}$ and $P_{2}=-7.319 \cdot 10^{-5}$. These parameters are used to correct radial and tangential image distortion, see for example [39]. The camera is connected to a laptop onboard the robot (Intel ${ }^{\circledR}$ Core $^{T M} 2$ Duo CPU at $2.50 \mathrm{GHz}$ ) with operating system Debian Linux. The observed target consists in a squared pattern, where the corners of the squares are extracted and matched to estimate the homography relating the current and target images. The acquired image data is processed using the OpenCV library ${ }^{1}$. The velocities computed by the control scheme are sent to the robot, and the control loop runs currently at $6.5 \mathrm{~Hz}$. The program communicates with the robot through the serial port using the ARIA library ${ }^{2}$ (from ActivMedia Robotics).

The results of a real experiment are shown in Fig. 12. The initial location is in region $\mathrm{V}$ and consists on a lateral

${ }^{1}$ http://www.intel.com/technology/computing/opencv

${ }^{2}$ http://www.activrobots.com/SOFTWARE/aria.html 


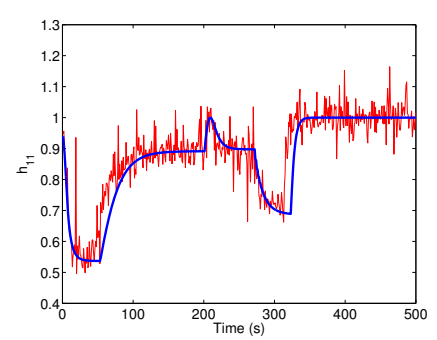

(a) Evolution of $h_{11}$

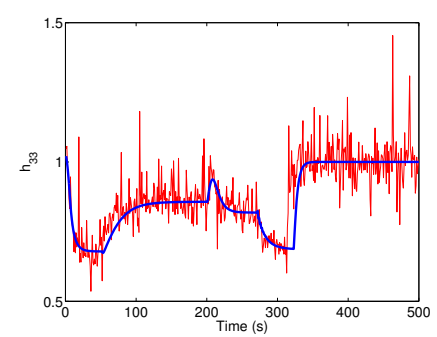

(c) Evolution of $h_{33}$

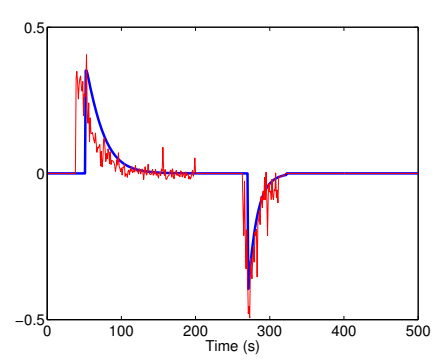

(e) Rotational control error

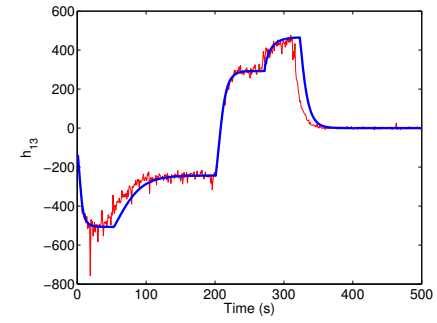

(b) Evolution of $h_{13}$

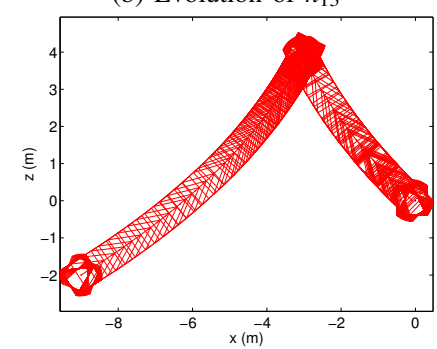

(d) Path with noise

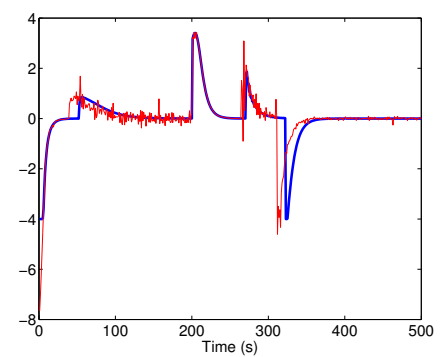

(f) Translational control error
Fig. 8. T-curves with and without image noise of $\sigma=1$ pixel. The initial location is $(-9,-2,-20)$ with observed target in $(x, z)=(-3,8)$.

translation of $0.8 \mathrm{~m}$ from the goal location with the same orientation. The resulting evolution of the homography entries and the computed velocities are depicted in Fig. 12, showing good behavior and that the signals remain well bounded and relatively smooth. A final error position error of several centimeters occurs in the real experiments. This is due to the system calibration errors and supports the simulated evaluation previously discussed. Additional real experiments are given in the video 2 and video 3 attachments. These videos show examples of a straight-line path starting in region I and a path of T-curves starting in region $\mathrm{V}$.

\section{CONCLUSIONS}

We have presented a visual control scheme intended for differential drive vehicles. The framework gives optimal trajectories taking into account the robot and the camera fieldof-view constraints. The control scheme presented drives the robot along these optimal paths from any position on the entire space to the goal position. This is a homographybased approach where the control is defined directly using the homography elements. Controllability analysis of the robot system constrained to drive on optimal paths is presented. The controllability of the homography-based control scheme and the stability according to Lyapunov are also studied. The feasibility of the proposed scheme has been demonstrated through simulations and real experiments with a robotic platform.

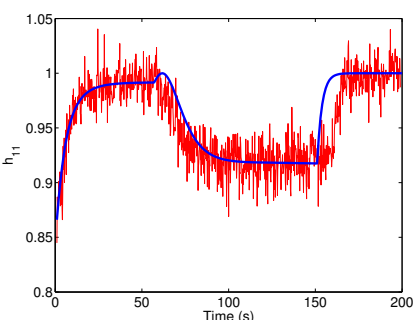

(a) Evolution of $h_{11}$

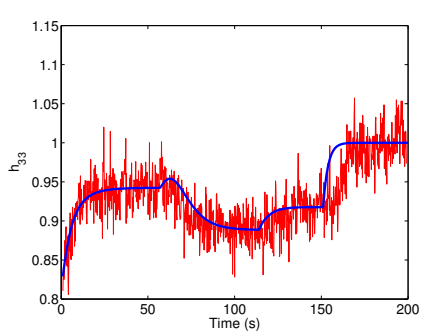

(c) Evolution of $h_{33}$

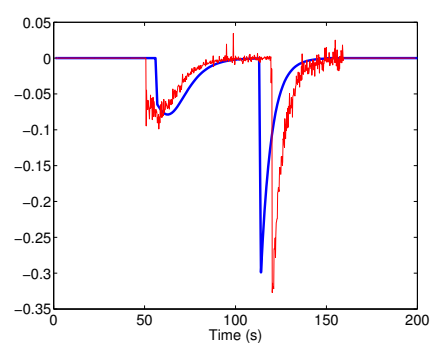

(e) Rotational control error

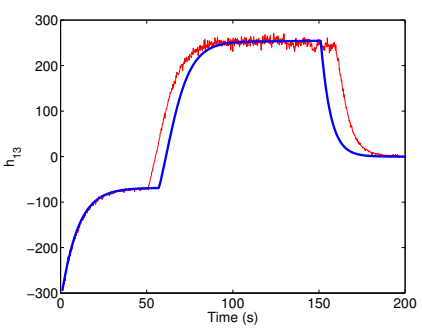

(b) Evolution of $h_{13}$

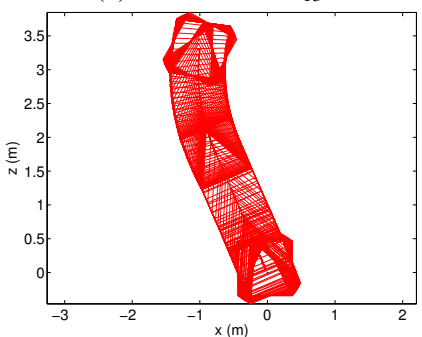

(d) Path with noise

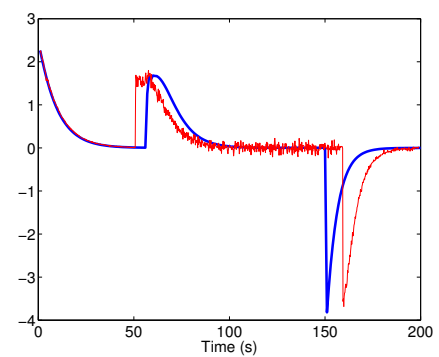

(f) Translational control error
Fig. 9. Simulations following a T-curve plus a SL path with and without image noise of $\sigma=1$ pixel. The initial location is $(-1,3.3,-20)$ with observed target in $(x, z)=(0.3,4.3)$.

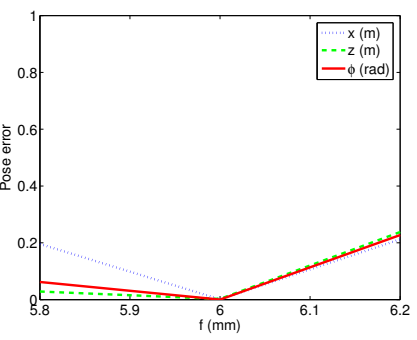

(a) SL path vs $f$

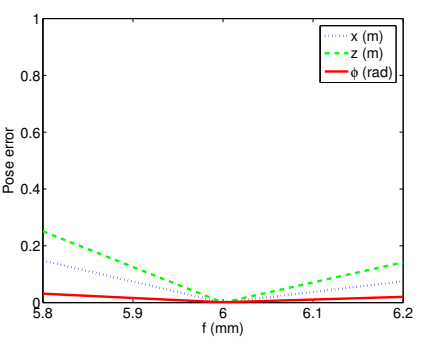

(c) T-curve path vs $f$

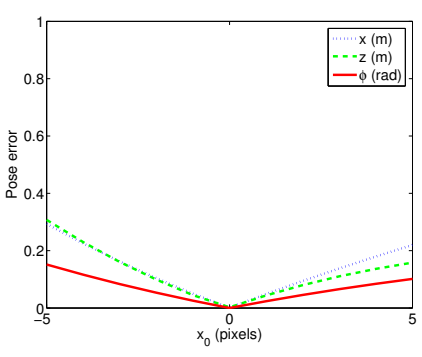

(b) SL path vs $x_{0}$

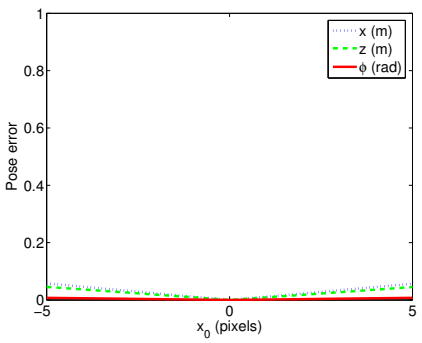

(d) T-curve path vs $x_{0}$
Fig. 10. Final position error with calibration errors for straight-line paths $(\mathrm{a}, \mathrm{b})$ and T-curve paths $(\mathrm{c}, \mathrm{d})$. The distance covered is around 10 meters. 


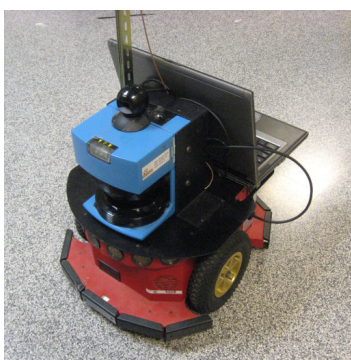

Fig. 11. The experimental platform with a camera and laptop onboard.

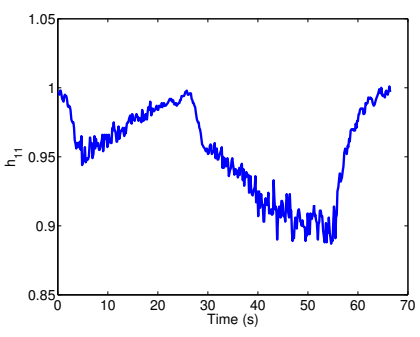

(a) Evolution of $h_{11}$

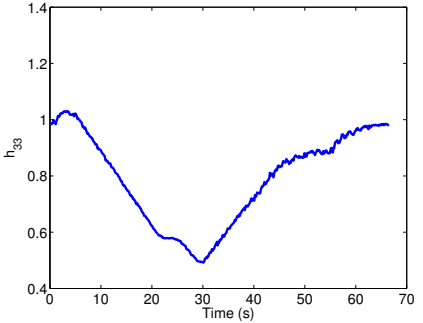

(c) Evolution of $h_{33}$

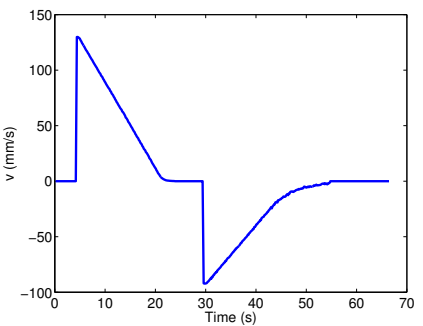

(e) Linear velocity $v$

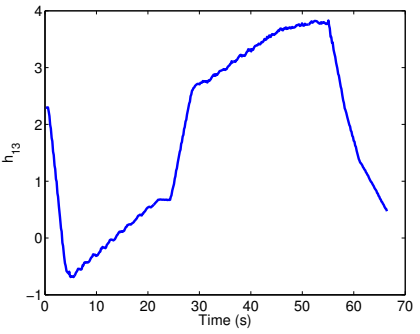

(b) Evolution of $h_{13}$

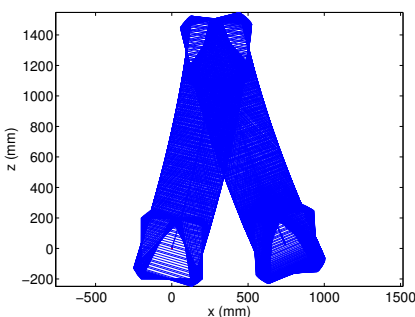

(d) Path

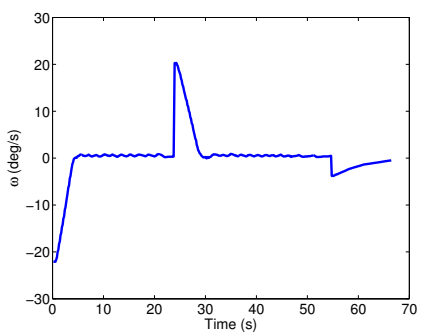

(f) Angular velocity $\omega$
Fig. 12. Real experiment with the initial location in region V. (a-c) Evolution of the homography entries $h_{11}, h_{13}$ and $h_{33}$, respectively. (d) Resultant path of the robot given by the odometry. (e-f) Velocities $(v, \omega)$ computed by the control scheme.

\section{ACKNOWLEDGMENT}

The authors would like to thank the anonymous reviewers for their insightful comments and helpful suggestions. This work was supported by projects DPI2006-07928, DPI200908126 and DPI2009-14664. This research was performed, in part, while N. Gans held a National Research Council Research Associateship Award at the Air Force Research Laboratory.

\section{REFERENCES}

[1] E. Malis, F. Chaumette, and S. Boudet, "2 1/2 D visual servoing," IEEE Trans. on Robotics and Automation, vol. 15, no. 2, pp. 234-246, 1999.
[2] S. Bhattacharya, R. Murrieta-Cid, and S. Hutchinson, "Optimal paths for landmark-based navigation by differential-drive vehicles with fieldof-view constraints," IEEE Transactions on Robotics, vol. 23, no. 1, pp. 47-59, 2007.

[3] S. Hutchinson, G. Hager, and P. Corke, "A tutorial on visual servo control," IEEE Trans. on Robotics and Automation, vol. 12, no. 5, pp. 651-670, 1996.

[4] G. DeSouza and A. Kak, "Vision for mobile robot navigation: A survey," IEEE Transactions on Pattern Analysis and Machine Intelligence, vol. 24, no. 2, pp. 237-267, 2002.

[5] J. Feddema and O. Mitchell, "Vision-guided servoing with feature-based trajectory generation," IEEE Trans. on Robotics and Automation, vol. 5, no. 5, pp. 691-700, Oct. 1989.

[6] B. Espiau, F. Chaumette, and P. Rives, "A new approach to visual servoing in robotics," IEEE Trans. on Robotics and Automation, vol. 8, no. 3, pp. 313-326, Jun. 1992.

[7] W. Wilson, C. Hulls, and G. Bell, "Relative end-effector control using cartesian position based visual servoing," IEEE Trans. on Robotics and Automation, vol. 12, no. 5, pp. 684-696, Oct. 1996.

[8] Y. K. Yu, K. H. Wong, M. M. Y. Chang, and S. H. Or, "Recursive camera-motion estimation with the trifocal tensor," IEEE Transactions on Systems, Man, and Cybernetics, Part B, vol. 36, no. 5, pp. 1081-1090, Oct. 2006.

[9] P. I. Corke and S. A. Hutchinson, "A new partitioned approach to image-based visual servo control," IEEE Transactions on Robotics and Automation, vol. 17, no. 4, pp. 507-515, August 2001.

[10] F. Chaumette and S. Hutchinson, "Visual servo control, part I: Basic approaches," IEEE Robotics and Automation Magazine, vol. 13, no. 4, pp. 82-90, Dec. 2006

[11] - "Visual servo control, part II: Advanced approaches," IEEE Robotics and Automation Magazine, vol. 14, no. 1, pp. 109-118, Mar. 2007.

[12] R. Basri, E. Rivlin, and I. Shimshoni, "Visual homing: Surfing on the epipoles," Int. J. of Computer Vision, vol. 33, no. 2, pp. 117-137, 1999.

[13] P. Rives, "Visual servoing based on epipolar geometry," in IEEE/RSJ International Conference on Intelligent Robots and Systems, vol. 1, 2000, pp. 602-607.

[14] G. L. Mariottini, G. Oriolo, and D. Prattichizzo, "Image-based visual servoing for nonholonomic mobile robots using epipolar geometry," IEEE Transactions on Robotics, vol. 23, no. 1, pp. 87-100, 2007.

[15] G. López-Nicolás, C. Sagüés, J. J. Guerrero, D. Kragic, and P. Jensfelt, "Switching visual control based on epipoles for mobile robots," Robotics and Autonomous Systems, vol. 56, no. 7, pp. 592-603, 2008.

[16] S. Benhimane and E. Malis, "Homography-based 2D visual servoing," in IEEE Int. Conf. on Robotics and Automation, 2006, pp. 2397-2402.

[17] S. Benhimane, E. Malis, P. Rives, and J. R. Azinheira, "Vision-based control for car platooning using homography decomposition," in IEEE Int. Conf. on Robotics and Automation, April 2005, pp. 2173-2178.

[18] C. Sagüés and J. J. Guerrero, "Visual correction for mobile robot homing," Robotics and Autonomous Systems, vol. 50, no. 1, pp. 41-49, 2005.

[19] Y. Fang, W. Dixon, D. Dawson, and P. Chawda, "Homography-based visual servo regulation of mobile robots," IEEE Transactions on Systems, Man, and Cybernetics, Part B, vol. 35, no. 5, pp. 1041-1050, 2005.

[20] J. Chen, W. Dixon, M. Dawson, and M. McIntyre, "Homographybased visual servo tracking control of a wheeled mobile robot," IEEE Transactions on Robotics, vol. 22, no. 2, pp. 406-415, April 2006.

[21] J. P. Laumond, Robot Motion Planning and Control. Springer Verlag, 1998.

[22] G. López-Nicolás, S. Bhattacharya, J. J. Guerrero, C. Sagüés, and S. Hutchinson, "Switched homography-based visual control of differential drive vehicles with field-of-view constraints," in IEEE International Conference on Robotics and Automation, Apr. 2007, pp. 4238-4244.

[23] N. Gans and S. Hutchinson, "A stable vision-based control scheme for nonholonomic vehicles to keep a landmark in the field of view," in IEEE Int. Conf. on Robotics and Automation, 2007, pp. 2196-2200.

[24] G. Kantor and A. Rizzi, "Feedback control of underactuated systems via sequential composition: Visually guided control of a unicycle," in Proc. of 11th Int. Symposium of Robotics Research, 2003.

[25] P. Murrieri, D. Fontanelli, and A. Bicchi, "A hybrid-control approach to the parking problem of a wheeled vehicle using limited view-angle visual feedback," International Journal of Robotics Research, vol. 23, no. 4-5, pp. 437-448, April-May 2004.

[26] O. D. Faugeras and F. Lustman, "Motion and structure from motion in a piecewise planar environment," Int. J. Pattern Recog. and Artificial Intell., vol. 2, no. 3, pp. 485-508, 1988. 
[27] R. I. Hartley and A. Zisserman, Multiple View Geometry in Computer Vision, 2nd ed. Cambridge University Press, 2004.

[28] G. López-Nicolás, C. Sagüés, and J. J. Guerrero, Shortest Path Homography-Based Visual Control for Differential Drive Robots. ITech Education and Publishing, Austria: G. Obinata and A. Dutta Eds. Vision Systems, 2007, ch. 30, pp. 583-596.

[29] D. J. Balkcom and M. T. Mason, "Time optimal trajectories for differential drive vehicles," International Journal of Robotics Research, vol. 21, no. 3, pp. 199-217, Mar. 2002.

[30] J. Weng, T. S. Huang, and N. Ahuja, Motion and Structure from Image Sequences. Berlin-Heidelberg: Springer-Verlag, 1993.

[31] J. A. Purcell, Calculus with Analytic Geometry. Prentice-Hall, Inc. Englewood Cliffs, NJ, 1978

[32] S. M. LaValle, Planning Algorithms. [Online], 2004, available at http://msl.cs.uiuc.edu/planning/.

[33] J. P. Laumond and J. J. Risler, "Nonholonomic systems: controllability and complexity," Theoretical Computer Science, vol. 157, pp. 101-114, 1996.

[34] H. Choset, K. M. Lynch, S. Hutchinson, G. Kantor, W. Burgard, L. E. Kavraki, and S. Thrun, Principles of Robot Motion: Theory, Algorithms, and Implementations. Boston: MIT Press, 2005.

[35] H. K. Khalil, Nonlinear Systems, 3rd ed. Prentice Hall, 2001.

[36] J. P. LaSalle, "An invariance principle in the theory of stability," in Differential Equations and Dynamical Systems, J. K. Hale and J. P. LaSalle, Eds. Academic Press, New York, 1967, pp. 277-286.

[37] J. H. Hespanha, "Uniform stability of switched linear systems: Extensions of LaSalle's invariance principle," IEEE Transactions on Automatic Control, vol. 49, no. 4, pp. 470-482, Apr. 2004.

[38] A. Bacciotti and L. Mazzi, "An invariance principle for nonlinear switched systems," Systems \& Control Letters, vol. 54, no. 11, pp. 1109 $-1119,2005$

[39] D. C. Brown, "Close-range camera calibration," Photogrammetric Engineering, vol. 37, no. 8, pp. 855-866, 1971. 